\title{
Adiciones a la flora liquénica foliícola de Argentina, Paraguay Oriental y regiones limítrofes de Brasil
}

\author{
Lidia Itati Ferraro* y Robert Lücking**
}

\author{
*IBONE, c.c. 209, 3400 Corrientes, Argentina \\ ** Lehrstuhl für Pflanzensystematik, Universität Bayreuth, D-95447 Bayreuth, Alemania.
}

\begin{abstract}
In the present paper the authors update the taxonomic and floristic knowledge of foliicolous lichenized fungi in northern Argentina and adjacent areas in Paraguay and southern Brazil. Twenty-seven taxa are reported for the first time, and the genera Phyllobathelium and Psorotheciopsis are recorded for the first time in Argentina. Furthermore, the new combination Gyalectidium catenulatum $\left[{ }^{\circ}\right.$ Tauromyces catenulatus] is proposed.

Resúmen: En este trabajo, los autores actualizan el conocimiento taxonómico y florístico de hongos liquenizados foliícolas para el norte de Argentina y áreas adjacentes en Paraguay y el sur de Brasil. Se agregan 27 taxones novedosos, y se mencionan por primera vez para Argentina los géneros Phyllobathelium y Psorotheciopsis. Además, se propone la nueva combinación Gyalectidium catenulatum [Tauromyces catenulatus].
\end{abstract}

\section{Introducción}

El registro actual para el área de estudio alcanza un número aproximado de 170 especies foliícolas, la mayoría de las colecciones han sido realizadas en el $\mathrm{N}$ argentino. Es interesante comparar estos números con los citados para otras regiones. Lücking et al. (2000) consideran que existen unas 730 especies de líquenes foliícolas en el mundo, la región que estudiamos representaría un $23 \%$ de ese total. En países húmedos tropicales con un gradiente altitudinal como Costa Rica, Colombia y Ecuador se calculan unas 350-400 especies (Lücking, 1999). En la Mata Atlántica de Pernambuco, un bosque perennifolio con estación seca marcada, en el nordeste de Brasil, se encontraron un total de 270 especies de líquenes foliícolas (Cáceres et al., 2000). Estos porcentajes indican que las localidades del NE-NW de Argentina y localidades limítrofes de Paraguay y Brasil, a pesar de su carácter subtropical y parcialmente seco, presentan una considerablemente alta diversidad de líquenes foliícolas. 
En la Prov. de Misiones en localidades del Parque Nacional Iguazú hemos coleccionado el mayor número de especies, aproximadamente $134 \mathrm{del}$ total de 170. Las restantes fueron encontradas en localidades de las provincias de Corrientes, Chaco y Formosa en Argentina. En localidades con alta humedad y una selva generalmente poco alterada, como es el área del Parque Nacional Iguazú (Argentina, Brasil), sobre una hoja se pueden encontrar de cuatro a diez especies, casi siempre con briófitas, algas y hongos. También los especímenes son mejor desarrollados que los encontrados en selvas de Corrientes o Formosa bastante más secas. Las colecciones que proceden de la selva de los Yungas, una región fitogeográfica que Cabrera (1971) denomina provincia de La Yunga, donde la vegetación predominante es la selva nublada, son importantes, ya que la flora liquénica foliícola, allí presente, es casi desconocida. Por el momento se realizaron colecciones en localidades de Jujuy y Salta, en laderas de cerros hasta una altura de $550 \mathrm{~m} \mathrm{~s} / \mathrm{m}$, se trata de una selva de transición con numerosos elementos de la llamada Provincia Chaqueña.

Se coleccionaron 230 ejemplares en total, de los cuales 65 corresponden a taxones foliícolas. Predominan familias como: Gomphillaceae, especialmente el género Gyalectidium con 4 especies (1 en estudio) y Calenia con 2 especies; Pilocarpaceae con 5 especies de Fellhanera y 4 de Byssoloma; Trichotheliaceae con 2 especies de Trichothelium y con numerosas especies de Porina, muchas aún no identificadas.

\section{Material y métodos}

Las hojas, una vez coleccionadas, fueron procesadas secándolas con aire caliente y luego mantenidas en freezer durante una semana. Para evitar contaminación por insectos, colocamos naftalina en polvo dentro de los sobres de papel. Las localidades visitadas hasta el momento donde se coleccionaron líquenes foliícolas son las siguientes:

ARGENTINA: Formosa, dep. Capital, Ea. Guaycolec, sobre las márgenes del riacho Pilagá. Chaco, dep. La Leonesa, Depto. Bermejo. Corrientes, dep. Capital, San Cosme, San
Miguel, Ituzaingó, Esquina, San Martín. Misiones, dep. Guaraní, Iguazú y dep. Gral. Belgrano. Salta, dep. Orán. Jujuy, dep. San Martín. PARAGUAY: Dep. Amambay, Cordillera, Misiones, Itapúa, Presidente Hayes. BRASIL: Estados de Río Grande Do Sul, Santa Catarina y Paraná. Otros ejemplares coleccionados en localidades de los Estados de Sao Paulo, Minas Gerais y Mato Grosso también fueron estudiados, éstas colecciones fueron realizadas por L. Ferraro durante la reunión „Recollecting Vainio“ realizada en Caraca (Minas Gerais) en Septiembre de 1997.

Los ejemplares originales se encuentran en el herbario de líquenes CTES, duplicados fueron distribuídos a los herbarios de CLU, LG y herbarios privados de R. Lücking y A. Vezda. Las observaciones microscópicas se hicieron con un microscopio Zeiss y una lupa Wild M5, los dibujos de la figura 3 fueron realizados con cámara clara.

Los taxones se enumeran en órden alfabético y la nomenclatura utilizada es la propuesta por Santesson (1952), Farkas \& Sipman (1997), Matzer (1996), Lücking (1997) y Lücking et al. (1998, 2000).

\section{Las especies}

Asterothyrium anomalum Kalb \& Vezda (Ostropales: Asterothyriaceae)

Fig. 1, A.

Especie descrita para Brasil (San Pablo, Foz de Iguazú), se menciona por primera vez para el NE de Argentina y Paraguay Oriental. El talo es orbicular, blanco y muy nítido, los apotecios se disponen en número de 3 o 4 o solitarios y más o menos de posición central, primero inmersos y luego elevados sobre el talo, prominentes, con el margen con restos talinos triangulares y el disco castaño con tinte rojizo.

Material estudiado: ARGENTINA. Misiones, dep. Iguazú, Parque Nacional Iguazú, límite E del Parque, 1200 mts. del río Iguazú, sobre hojas de Bignoniaceae, 11-VIII-95, Vanni et al. 3528 (CTES); ídem, sendero Yacaratia, sobre hojas de 

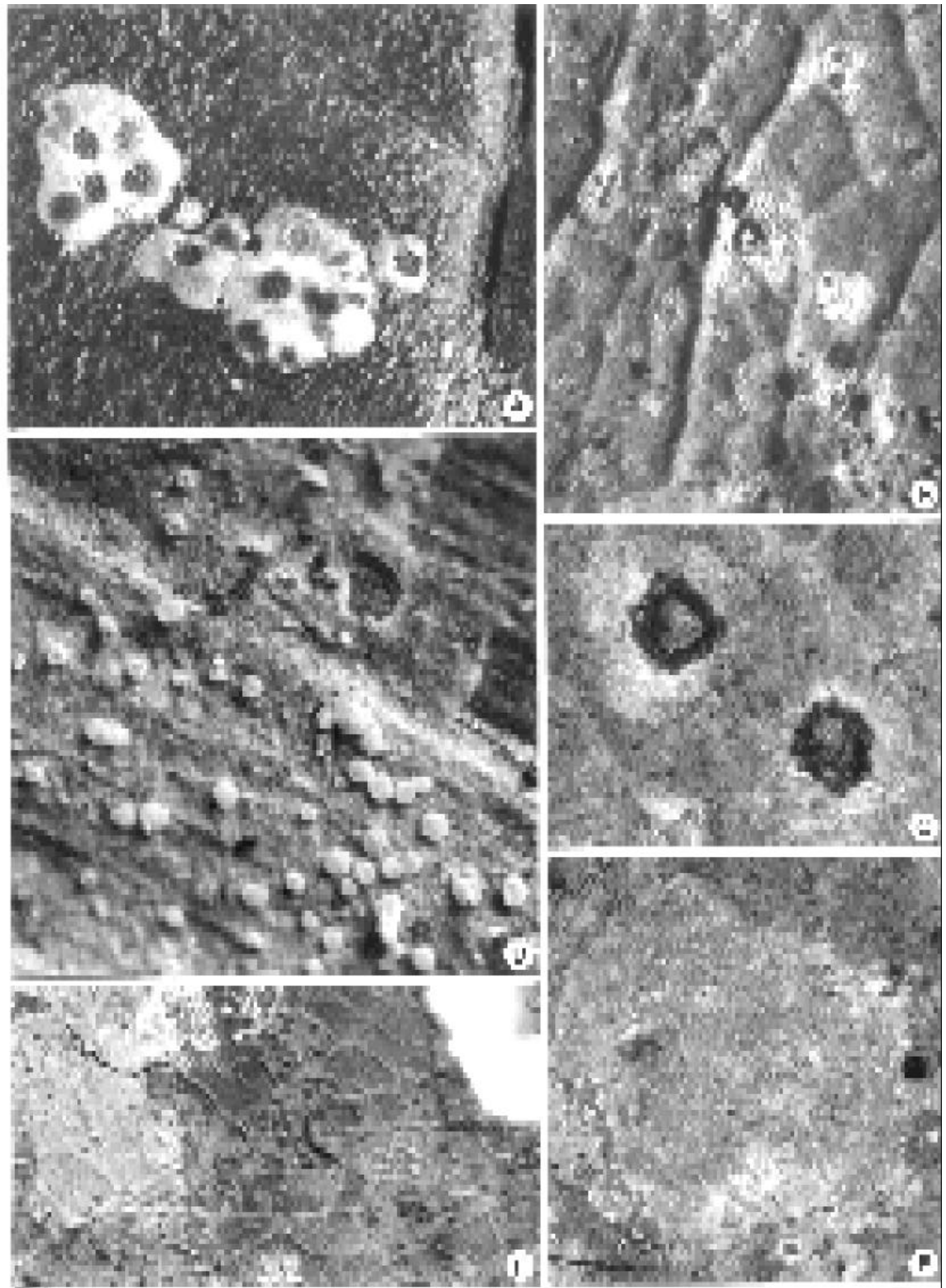

Fig. 1: A, Asterothyrium anomalum (Maruñak 900, CTES); B-C, Aulaxina quadrangula (Popoff 2887, CTES); D, Dimerella minima (Maruñak 910, CTES); E, Echinoplaca handelii (Popoff 2890, CTES); F, Fellhanera parvula (Ferraro 5015, CTES).

TROPICAL BRYOLOGY 19 (2000) 
Sorocea bonplandii (Moraceae), 8-VIII-95, Maruñak et al. 900 (CTES); dep. Guaraní, Picada de límite del Predio desde ruta ${ }^{\circ} 15$ al E, 1500 $\mathrm{m}$, sobre hojas de Cupania vernalis, 8-IX-94, Maruñak et al. 744 a (CTES, ULM, det. R. Lücking); dep. Gral. Belgrano, Colonia Andresito, Chacra 30, 1-III-96, Vanni et al. 3654 (CTES).

Aulaxina quadrangula (Stirt.) R. Sant. (Ostropales: Gomphillaceae)

Fig. 1, B-C

Común en Misiones. Fácil de identificar por sus apotecios angulosos con margen muy oscuro o negro, prominentes. Se la registraba para Ecuador, Perú, Brasil, Bolivia (Santesson, 1952), Costa Rica (Lücking, 1992) y ahora agregamos las colecciones de Argentina.

Material estudiado: ARGENTINA. Misiones, dep. Iguazú, Parque Nacional Iguazú, sendero Macuco, 4-III-96, Popoff 2887 (CTES, hb. Vezda).

Bapalmuia palmularis (Müll. Arg.) Sérus. (Lecanorales: Pilocarpaceae)

Material estudiado: Argentina. Misiones, dep. Guaraní, Predio Guaraní, picada del límite del Predio desde ruta 15 al E, 1500 mts., sobre Nectandra megapotamica (Lauraceae), 8-IX-94, Maruñak 708 (CTES).

Byssolecania deplanata (Müll. Arg.) R. Sant. (Lecanorales: Pilocarpaceae)

Especie común en el NE de Argentina con apotecios muy planos y fuertemente adnatos, castaños y cuando adultos despegados del talo, esporas oblongas con 5-7 septos.

Se trata de una especie que ya se había mencionado para Brasil, Costa Rica y Afríca. En el herbario CTES existen tambien colecciones realizadas por A. Krapovickas ( $n^{\circ} 46769$ y 46788) en Bolivia, dep. La Paz, son los ejemplares más típicos que hemos estudiado.
Material estudiado: ARGENTINA. Misiones, dep. Iguazú, Parque Nacional Iguazú, sendero Yacaratia, 8-VIII-95, Maruñak et al. 893 (CTES, hb. Lücking); ídem, sobre hojas de Rhapanea, Ferraro, Maruñak y Vanni 5072 (CTES); ídem, sendero Macuco, sobre hojas de Sorocea, Ferraro, Maruñak y Vanni 5042 (CTES); ídem, Ruta 101, arroyo Santo Domingo, sobre hojas de Sorocea, Ferraro, Maruñak y Vanni 5164 (CTES, hb. Lücking).

Byssoloma chlorinum (Vain.) Zahlbr. (Lecanorales: Pilocarpaceae)

Previamente registrado para Paraguay (Ferraro 1997), los materiales que citamos constituyen las primeras menciones para Argentina.

Material estudiado: ARGENTINA. Corrientes, dep. San Cosme, Paso de La Patria, en quebrachal degradado, sobre hojas de Oncidium, 16-I-93, Ferraro 4562 (CTES). Misiones, dep. Iguazú, Parque Nacional Iguazú, límite E del Parque, 1200 m del río Iguazú, 11-VIII-95, Vanni et al. 3523 (CTES); dep. Guaraní, Picada de límite del Predio desde ruta $\mathrm{n}^{\circ} 15$ al E, $1500 \mathrm{~m}$, sobre hojas de Pouteria salicifolia (Sapotaceae), 8-IX-94, Maruñak 759 (CTES).

\section{Byssoloma minutissimum Kalb \& Vezda}

Las colecciones de esta especie son las primeras para Argentina, era conocida de Brasil, Costa Rica y Guatemala. En el herbario CTES existen colectas realizadas por A. Schinini en

Mato Grosso, Brasil.

Material estudiado: Argentina. Misiones, dep. Guaraní, Predio Guaraní, 26 54'-59'S, 54 12'$18^{\prime} \mathrm{W}$, Sendero CIFOR, sobre hojas de Merostachys (Gramineae), 27-IV-99, Ferraro, Tressens, Keller, Maruñak y Revilla 6031 (CTES).

Calopadia subcoerulescens (Zahlbr.) Vezda (Lecanorales: Ectolechiaceae)

Se conocen otras 4 especies del género en 
Argentina, Calopadia foliicola, C. fusca, C. phyllogena y C. puiggarii.

Material estudiado: Argentina. Salta, dep. Orán, Finca San Andrés, en Yungas, selva secundaria, 28-X-97, Schinini 34213a y 34216 (CTES).

Chroodiscus coccineus (Leight.) Müll. Arg. (Ostropales: Thelotremataceae)

Especie muy llamativa por sus apotecios anaranjado brillantes y el talo verde con verrugas grandes.

Si bien el género comprende 8 especies foliícolas, hasta el momento es la única que coleccionamos. Chroodiscus coccineus es común en selvas de América tropical, fue registrada también en Costa Rica, Perú, Brasil y Bolivia .

Material estudiado: ARGENTINA. Misiones, dep. Iguazú, Parque Nacional Iguazú, sendero Yacaratia, 8-VIII-95, Maruñak et al. 883 (CTES, Hb. Lücking).

Dimerella minima (Müll. Arg.) R. Sant. (Gyalectales: Gyalectaceae)

Fig. 1, D

El registro de esta especie es importante ya que era conocida solo de Costa Rica (Santesson, 1952; Lücking, 1992). El ejemplar de la provincia de Misiones que mencionamos crece sobre hojas de Sorocea bonplandii. Se trata de una especie con talo inconspicuo, apotecios globosos, con el disco levemente urceolado, amarillentos, muy pequeños, con margen prominente y esporas 12 15 x 3-4 um.

Material estudiado: ARGENTINA. Misiones, depto. Iguazú, Parque Nacional Iguazú, sendero Yacaratia, 8-VIII-95, Maruñak et al. 910 (CTES).

Echinoplaca epiphylla Fée (Ostropales: Gomphillaceae)
Es la especie mas representativa del género en el área de estudio. Caracterizada por sus apotecios planos, amarillentos y el talo verrucoso provisto de pelos estériles.

Material estudiado: Argentina. Formosa, dep. Capital, Ea. Guaycolec, 22 Kms N de Formosa, ruta 11 , en borde de selva marginal del riacho Pilagá, sobre hojas de Pilocarpus, 15-II-95, Ferraro, López y Vanni 4608 (CTES). Misiones, dep. Iguazú, Parque Nacional Iguazú, paseos inferiores, 29-II-96, Vanni et al. 3630 (CTES).

\section{Echinoplaca handelii (Zahlbr.) Lücking}

Fig. 1, E

En esta especie el talo es verrucoso, puede tener pelos blancos estériles, que no están presentes en el ejemplar de Misiones que ilustramos. Los apotecios son bastante planos, adnatos, castañoclaro hasta amarillentos, generalmente brillantes, sin margen. Las esporas oblongas varían en número de 4 a 6 en el asco y son submuriformes. Los apotecios adultos pueden elevarse en la región central.

Había sido mencionada con anterioridad para Brasil, Costa Rica y China.

Material estudiado: ARGENTINA. Misiones, dep. Iguazú, Parque Nacional Iguazú, sendero Macuco, 4-III-96, Popoff 2890 (CTES).

Fellhanera emarginata Lücking (Lecanorales: Pilocarpaceae)

Esta especie fue descrita para Costa Rica, el ejemplar que estudiamos para el NE de Argentina constituye el primer registro para el área. Presenta apotecios castaño-amarillentos, sin margen y esporas con 3 septos.

Material estudiado: ARGENTINA. Misiones, dep. Guaraní, Predio Guaraní, picada del límite del Predio, desde ruta 15 al E, $1500 \mathrm{~m}$, sobre hojas de Olyra (Gramineae), 8-IX-94, Maruñak et al. 688 (CTES, hb. Lücking). 


\section{Fellhanera paradoxa (Vezda) Vezda}

Se caracteriza por los apotecios castaño-oscuro, con margen prominentes y esporas anchas con 3 septos o irregularmente septadas hasta submuriformes en el mismo himenio (Lücking, 1997). La mayoría de los ejemplares mencionados en este trabajo tienen esporas con 3 septos, aunque unos pocos presentan 4 y 5 septos. Puede confundirse externamente con Fellhanera fuscatula, una especie que tiene apotecios con margen delgado y esporas 7 septadas.

Fellhanera paradoxa es una especie común en el área de estudio, podemos decir que junto con $F$. bouteillei y $F$. semecarpi son las mejor representadas de este complicado grupo.

Material estudiado: ARGENTINA. Misiones, dep. Guaraní, Predio Guaraní, picada del límite del Predio, desde ruta 15 al E, $1500 \mathrm{~m}$, sobre hojas de Piper gaudichanum, 8-IX-94, Maruñak et al. 728 (CTES, hb. Lücking); ídem, sobre hojas de Corymborchis flava, Maruñak 756 (CTES); ídem, Maruñak 814 (CTES, LG); dep. Iguazú, Parque Nacional Iguazú, sendero Macuco, sobre hojas de Rutaceae, Ferraro et al. 4999, 5011 y 5019 (CTES); sendero Yacaratia, sobre hojas de Rhapanea, Ferraro et al. 5073 (CTES, hb. Lücking, CLU) 29-III-95; ídem, sobre hojas de Actinostemon, Ferraro et al. 5091 (CTES); idem. sobre hojas de Psychotria, Ferraro et al. 5096 (CTES, hb. Vezda); ídem. Isla Grande frente al Puerto Canoas, en selva sobre hojas de Sorocea, Ferraro et al. 5152 (CTES). Formosa, dep. Capital, Estancia Guaycolec, $22 \mathrm{Kms} \mathrm{N}$ de Formosa, ruta 11, en borde de selva marginal del riacho Pilagá, sobre hojas de Nectandra, 15-II95, Ferraro et al. 4636 (CTES, CLU).

\section{Fellhanera parvula (Vezda) Vezda}

Fig. 1, F

Con apotecios de color pálido castaño o amarillentos y muy pequeños, esporas con 1 septo.

Material estudiado: ARGENTINA. Misiones, dep. Iguazú, Parque Nacional Iguazú, sendero
Macuco, Ferraro et al. 5015 (CTES, hb. Lücking).

\section{Fellhanera vandenberghenii (Sérus.) Vezda}

Fellhanera vandenberghenii tiene talo muy granuloso, esporas con un septo y picnidios en forma de frasco o botella, con el cuello bastante alargado. Fue descrita de localidades del S de Africa como Dimerella vandenberghenii (Sérusiaux, 1983) Es afín a $F$. misionensis, una especie que habita selvas del NE argentino, de Paraguay y de Brasil, recientemente descrita (Ferraro \& Lücking, 1999). La presencia de los picnidios es lo que permite identificar a $F$. vandenberghenii ya que $F$. misionensis no presenta picnidios de este tipo.

Material estudiado: ARGENTINA. Misiones, dep. Guaraní, Picada del límite del predio desde ruta 15 al $\mathrm{E}, 1500 \mathrm{~m}$, sobre hojas de Bignoniaceae, 8-IX-94, Maruñak 731 (CTES, hb. Lücking). PARAGUAY, Misiones, W de la Isla Yacyretá, sobre hojas de Plinia rivularis (Myrtaceae), 25-XI-88, Ferraro et al. 3680 (CTES, hb. Vezda).

\section{Gyalectidium (Ostropales: Gomphillaceae)}

Este género comprende en la actualidad 18 especies con distribución mundial, la mayoría presentes en la región que estudiamos. Lücking et al. (1998) han revisado las colecciones de hongos foliícolas liquenizados, que Batista y colaboradores realizaran en Brasil y han encontrado especies de Gyalectidium bajo otros géneros. De localidades de Argentina y Paraguay proceden 4 especies nuevas para la ciencia que están siendo descritas. Esta región de América del Sur parece ser un punto geográfico importante en la dispersión de estos taxones. En esta contribución se propone la nueva combinación:

Gyalectidium catenulatum (Caval. \& Silva) Ferraro, Lücking \& Sérus. comb. nov.

Fig. 2, A 

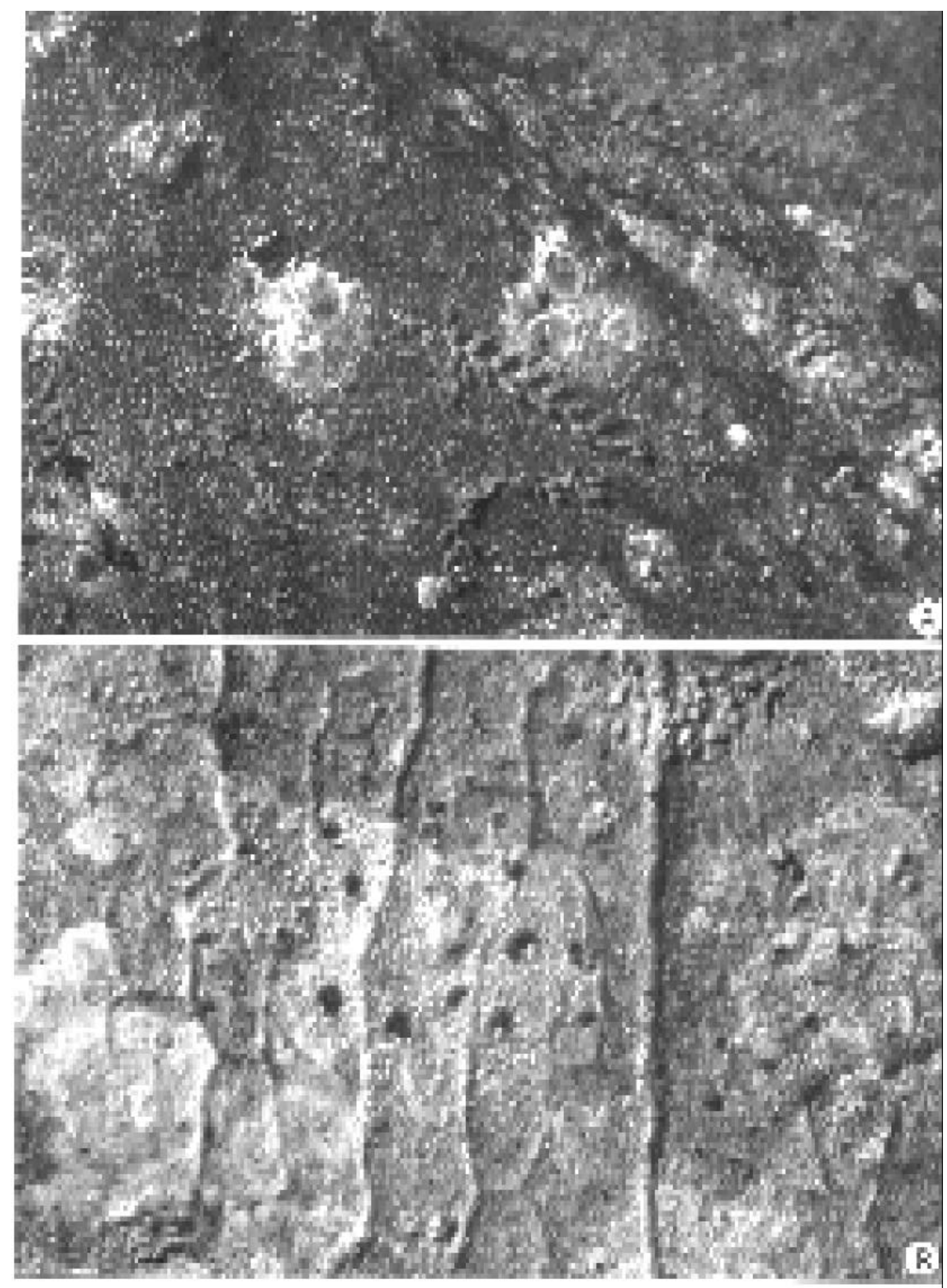

Fig. 2: A, Gyalectidium catenulatum (Vanni 3652, CTES); B, G. imperfectum (Ferraro 6043, CTES). 
Basónimo: Tauromyces catenulatus Caval. \& A. A. Silva, in Cavalcante et al., Publcões Inst. Micol. Recife, 647: 37 (1972)

Talo 4-5,5 mm diám., brillante, delgado y usualmente liso hasta algo irregular, verrucoso por la acumulación de cristales en algunas zonas, verrugas dispersas, pequeñas, borde talino ondulado con hipotalo transparente, muy claro, adherido al sustrato, a veces desprendido en partes, 95-100 um alt en corte. Manchas talinas aisladas, con 1 hifóforo y 3-5 apotecios.

Hifóforos del tipo escamoso o tipo Gyalectidium filicinum, un poco mas largos hasta $0,5 \mathrm{~mm}$ long x 0,1-0,2 mm anchos, escamas algo acanaladas con prolongaciones laterales cortas, generalmente dispuestos en el margen del talo.

Apotecios siempre presentes, planos, anchos, 0,30,35 mm diám., con un margen rojizo o castañooscuro levemente elevado, a veces concoloro con el disco; disco verde-grisáceo, blanquecino, ceniciento (malva) a verde-claro algo hundidos con respecto al margen.

Ascos globosos con paredes gruesas, con las bases adelgazadas, algo clavadas. Espora única, muriforme, 23(27)-29 um x 8-14 um, incoloras, ocupando completamente el asco.

Células algales globosas, verde intenso, 9-10 um diám.

Observaciones: Cavalcante \& Silva (1972) identifican y describen para Brasil Tauromyces, basado en Tauromyces catenulatus, género considerado sinónimo de Gyalectidium (Farkas \& Sipman, 1993). T. catenulatus se menciona a su vez como sinónimo de G. filicinum (Sérusiaux, 1992). Previamente a conocer Tauromyces catenulatus, los autores hemos estudiado material de Argentina perteneciente a Gyalectidium, que denominamos „G. laevigatum“ y „G. cucullatum“, ambos nombres no válidamente publicados. El nombre correcto para identificar al material de Argentina y Brasil sería Gyalectidium catenulatum (Cavalc. \& Silva) Ferraro, Lücking \& Sérus. Mientras que la sinonimia entre $T$. catenulatus con G. filicinum, no se considera. G. filicinum es una buena especie que no se involucra en la nueva combinación propuesta. Se trata de una especie muy abundante en nuestra región.
Material estudiado: ARGENTINA. Corrientes, dep. Capital, Perichón, sobre Myrtaceae, 12-X83, Benitez 312a (CTES); dep. Santo Tomé, Arroyo Chimiray, interior de selva, sobre Lauraceae, 23-IX-1974, Krapovickas et al. 36751 (CTES, LG). Misiones, dep. Guaraní, Picada de límite del Predio desde ruta n 15 al E, 1500 m, sobre hojas de Bignoniaceae, 8-IX-94, Maruñak 733 (CTES, CLU); ídem, sobre hojas de Sorocea bonplandii, 8-IX-94, Maruñak 729 (CTES); ídem, sobre hojas de Cupania vernalis, 8-IX-94, Maruñak 744 (CTES, hb. Lücking); ídem, acceso al arroyo Soberbio, foliícola sobre frondes de Pteris deflexa, 8-IX-94, Maruñak 823 (CTES); ídem, sobre Citronella paniculata, 8-IX-94, Maruñak 846 (CTES); Predio Guaraní, sobre hojas de Lonchocarpus (Leguminosae), 24-II-95, Tressens et al. 5511 (CTES); ídem, sobre hojas de Casearia silvestris (Flacourtiaceae), 24-II-95, Tressens et al. 5515 (CTES); dep. Iguazú, Parque Nacional Iguazú, sendero Jacaratia, 8-VIII-95, Maruñak 874 (CTES); ídem, Maruñak 898 (CTES); ídem, en selva de Myrtaceae, sobre hojas de Roupala cataractarum (Proteaceae), 9-VIII95, Maruñak 914 (CTES); Parque Nacional Iguazú, límite E del Parque, 1200 mts. del río Iguazú, 11-VIII-95, Vanni et al. 3526 (CTES); ídem, sendero Macuco, sobre hojas de Melastomataceae, 28-III-95, Ferraro et al. 5054 (CTES); ídem, sendero Jacaratia, sobre hojas de Piper, 29-III-95, Ferraro et al. 5062 (CTES); ídem, sobre hojas de Olyra, 29-III-95, Ferraro et al. 5066 (CTES); ídem, sobre hojas de Rhapanea, 29-III-95, Ferraro et al. 5069 (CTES); dep. Candelaria, Loreto, en islote de selva, sobre Lauraceae, 19-XII-81, Ferraro 2404 (CTES, hb. Lücking); dep. General Belgrano, Colonia Andresito, Chacra 30, sobre hojas de Guarea (Meliaceae), 1-III-96, Vanni, Kurtz, Popoff \& Herrera 3652 (CTES). Salta, dep. Orán, Finca San Andrés, en Yungas, selva secundaria, ca. $28^{\circ} 04^{\prime} 23^{\prime \prime}$ S, $64^{\circ} 45^{\prime} 07^{\prime \prime}$ W, 28X-97, Schinini 34213 (CTES). PARAGUAY. Misiones, W de la Isla Yacyretá, sobre hojas de Plinia rivularis (Myrtaceae), 25-XI-88, Ferraro et al. 3682 (CTES); dep. Canendiyú, $22 \mathrm{Kms}$ de Colonia Nueva Esperanza, Reserva Biológica Itabó, $24^{\circ} 35^{\prime}$ S, $54^{\circ} 48^{\prime} \mathrm{W}, 260$ m s.m., en selva primaria, tierra arcilloso-colorada, sobre hojas de Thelypteris, 15-X-96, Schinini et al. 31571 
(CTES); ídem, sobre hojas de Ilex, 15-X-96, Schinini et al. 31575 (CTES), ídem, huésped indet., 15-X-96, Schinini et al. 31581 (CTES). BRASIL. Río Grande Do Sul, Iraí, ladera selvática sobre el río Do Mel, Balneario Osvaldo Cruz, foliícola, sobre hojas de naranjo, 22-V-90, Cristóbal 2242 y 2243 (CTES, hb. Vezda), ídem, sobre hojas de Trichilia catigua, 29-I-92, Krapovickas y Cristóbal 44125 (CTES).

\section{Gyalectidium imperfectum Vezda}

Fig. 2, B

Especie bien representada en nuestra región. El talo es extendido, finamente verrucoso y presenta manchas oscuras, semilunares, correspondientes a los hifóforos; las masas de conidios no se encuentran en la axila de escamas, como ocurre en la mayoría de las especies de éste género. Los ejemplares jóvenes puedan confundirse con $G$. filicinum pero el talo con verrugas grandes de esta especie y los apotecios prominentes permiten diferenciarlas.

Material estudiado: ARGENTINA. Misiones, dep. Guaraní, Picada de límite del Predio desde ruta $\mathrm{n}^{\circ} 15$ al E, $1500 \mathrm{~m}$, sobre hojas de Olyra (Gramineae), 8-IX-94, Maruñak 690 (CTES, hb. Lücking), ídem, sobre hojas de Bignoniaceae, 8IX-94, Maruñak 735 (CTES), Predio Guaraní, sobre Lonchocarpus (Leguminosae), 24-II-94, Tressens et al. 5511(CTES), ídem, 5512 (CTES, LG); dep. Iguazú, Parque Nacional Iguazú, sendero Jacaratia, 8-VIII-95, Maruñak et al. 888 (CTES), ídem, Maruñak et al 905 (CTES), ídem, Maruñak et al 911 (CTES, hb. Vezda), sendero Macuco, sobre frondes de Doryopteris, 28-III95, Ferraro, Maruñak y Vanni 5028 (CTES), ídem, límite E del Parque Nacional Iguazú, 1200 $\mathrm{m}$ del río Iguazú, sobre helechos, 11-VIII-95, Vanni et al. 3513 (CTES). Dep. Guaraní, Predio Guaraní, sobre Casearia sp. (Flacourtiaceae), 24II-95, Tressens et al. 5513 (CTES). Formosa, dep. Capital, Estancia Guaycolec, sobre hojas de Sorocea sprucei, VII-89, Hb Palaccí-Arditi 85 (CTES). Salta, dep. Orán, Finca San Andrés, en yungas, selva secundaria, Schinini 34167 (CTES), ídem, „Estancia La Maroma“, 1000 m s.m., sobre hojas de Solanum symetricum
(Solanaceae), 21-VIII-98, Saravia Toledo s/n, Fundación para el Desarrollo del Chaco, Colección Norandino-Techint. Jujuy, dep. Gral. San Martín, Parque Nacional Calilegua, Sendero al Camping Aguas Negras, en selva, Distrito Yungas, sobre hojas de Trichilia hyeronimii (Meliaceae), 17-IV-1998, Ferraro et al. 5873 (CTES). PARAGUAY. Caazapá, Distrito Yuty, $15 \mathrm{Km}$. S de Capitindy, abra en la selva, borde de arroyo, pastizal inundable, sobre hojas de Actinostemun concolor, 30-IX-87, Arbo et al. 2937a (CTES). Presidente Hayes, cercanías de la „Estancia Maroma“, $25 \mathrm{Kms}$ E de Pozo Colorado, interior de bosque de Schinopsis balansae, sobre hojas de Capparis retusa, 10VI-95, Mereles y Degen s/n (CTES).

\section{Lyromma (Dothideales)}

Se trata de un género descrito para Brasil, que actualmente comprende 4 especies distribuídas en regiones tropicales y pantropicales del mundo. Durante mucho tiempo ha sido clasificado entre los líquenes imperfectos, pero desde que se hallaron ejemplares con ascos y esporas, se incluyen entre los Dothideales. En nuestro criterio se trata de un Ascomycete liquenizado, ya que la opinión de algunos autores es que no se trataría de un liquén. Los talos de las especies mencionadas tienen forma circular, de color verde brillante, con aspecto transparente, con algas del género Phycopeltis.

Es posible que el pequeño tamaño de las estructuras reproductivas justifique el escaso conocimiento de estos taxones, ya que generalmente pasan desapercibidos totalmente en las colecciones.

Las 3 especies que viven en Argentina han sido analizadas microscópicamente.

\section{Lyromma nectandrae Batista \& Maia}

Fig. 3, A. Fig. 4, A-D

Fue descrito para Brasil y mencionado por Lücking (1992) para Costa Rica. Hemos realizado el exámen microscópico de los ejemplares citados, en la mayoría solamente se observan picnidios. Los picnidios son castaño-oscuros y 


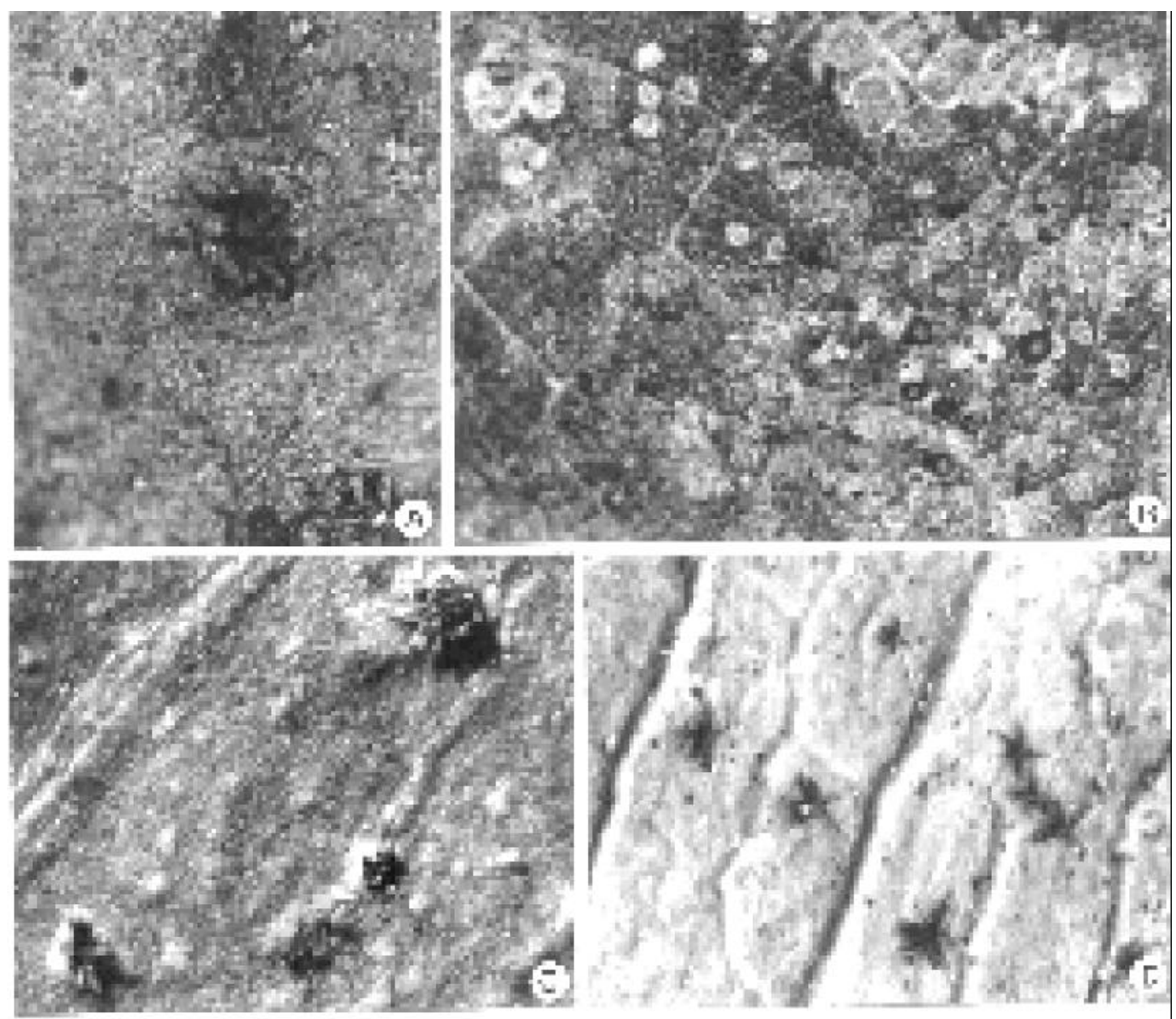

Fig. 3: A, Lyromma nectandrae (Ferraro 5916, CTES); B, Psorotheciopsis philippinensis (Ferraro 6174, CTES); C, Trichothelium ake-assii (Ferraro 5015, CTES); D, T. bipindense (Ferraro 5027, CTES).

tienen forma oblongo-alargada, en estado maduro presentan en su extremo 2 a 4 largas prolongaciones.

El ejemplar de Misiones (col. Popoff $n^{\circ} 2896$ ) es más típico en lo que respecta a la forma de los picnidios, coincidiendo con las características indicadas en las pocas descripciones sobre esta especie. Los conidios son filiformes, flexuosos y muy abundantes.

Esta especie fue coleccionada en el NE de Argentina en selvas húmedas en el área de las cataratas del Iguazú.

Material estudiado: ARGENTINA. Misiones, dep. Iguazú, Parque Nacional Iguazú, sendero Macuco, sobre hojas de naranjo, 4-3-1996, Popoff
2896 (CTES), ídem, Parque Nacional Iguazú, paseos inferiores, sobre Gaurea, 29-2-1996, Vanni et al. 3631 (CTES); dep. Guaraní, Predio Guaraní, 26 ${ }^{\circ}$ 54'-59'S, 54', 12'-18'W, sobre hojas de Actinostemon concolor, 27-IV-1999, Ferraro et al. 6056 (CTES, Hb. Lücking). Mato Grosso, Mun. Pontes e Lacerda, Rodovia BR174, prosemo a Corrego das Burjes, sobre helechos, 16-8-1997, Schinini 33996 (CTES).

Lyromma ornata Lücking, Kalb \& Sérus. Fig. 4, E

Los ejemplares de Jujuy (col. Ferraro no 5916) presentan peritecios con prolongaciones mas 
cortas, anchas en la base, mas o menos triangulares en el extremo y también en mayor número que las mencionadas para los picnidios, con ascos globosos muy pequeños y esporas cilíndricas con 3 septos. El material es bastante escaso. Lyromma ornata (Lücking \& Kalb, 2000 ) es una especie recientemente estudiada de localidades del Estado de São Paulo, Brasil.

Material estudiado: ARGENTINA. Jujuy, dep. General San Martín, Parque Nacional Calilegua, sendero al camping „Aguas Negras“, en selva, Distrito Yungas, sobre hojas de Trichilia hyeronimii (Meliaceae), 17-IV-1998, Ferraro y Popoff 5916 (CTES).

Lyromma palmae (Cavalc. \& A. A. Silva) Lücking \& Sérus.

Fig. 4, F

Esta especie fue coleccionada también para la provincia de Jujuy (NW de Argentina) en la selva de Los Yungas, junto a Lyromma ornata. El aspecto de los picnidios es muy similar a los descritos para L. nectandrae, un poco mas cortos $\mathrm{y}$ angostos y sin las prolongaciones corniculadas en el extremo, en general mas pequeños.

Material estudiado: ARGENTINA. Jujuy, dep. General San Martín, Parque Nacional Calilegua, sendero al camping „Aguas Negras“, en selva, Distrito Yungas, sobre hojas de Trichilia hyeronimii (Meliaceae), 17-IV-1998, Ferraro y Popoff 5916 (CTES).

Phyllobathelium firmum (Stirt.) Vezda (Pyrenulales: Strigulaceae)

Es la primera vez que se menciona el género para Argentina, Santesson (1952) lo menciona para Brasil y Ferraro (1997) registra material de Paraguay. Los ejemplares hallados son robustos y con abundantes fructificaciones. Lücking (1997) considera que este taxon es un elemento característico de la región Neotrópica.

Presenta verrugas periteciales, anchas, elevadas y cubiertas por el talo.
Material estudiado: ARGENTINA. Misiones, dep. Iguazú, Parque Nacional Iguazú, ruta 101, Maruñak et al. 857 (CTES) y Maruñak et al. 859 (CTES); ídem. sendero Yacaratia, sobre hojas de Sorocea bonplandii, 8-VIII-95, Maruñak et al. 878 (CTES).

Psorotheciopsis philippinensis (Rehm) Lücking (Ostropales: Asterothyriaceae)

Fig. 3, B

Se incluyen bajo este nombre ejemplares caracterizados por apotecios robustos, oscuros y esporas con un septo.

Si bien en este trabajo lo incluimos en la familia Asterothyriaceae, actualmente la posición taxonómica del género es discutida.

Material estudiado: ARGENTINA. Misiones, dpto. Guaraní, Predio Guaraní, ruta 15 hacia Papel Misionero, en bosque de Laurel, sobre hojas de Luhea (Tiliaceae), 28-IV-99, Ferraro et al. 6174 (CTES); ídem, sobre hojas de Schinus, Ferraro et al. 6169 (CTES).

Tapellaria phyllophila (Stirt.) R. Sant. (Lecanorales: Ectolechiaceae)

Con apotecios llamativos de hasta $1 \mathrm{~mm}$ de diám., negros, brillantes y con esporas submuriformes. Es una especie pantropical mencionada para Brasil por Santesson (1952) y Ferraro (1997). Se trata de una especie poco coleccionada, no siempre presente en los herbarios.

Material estudiado: ARGENTINA. Corrientes, dep. Santo Tomé, Ruta 37, 8 kms. E de Gobernador Virasoro, creciendo sobre hojas de Maytenus, Schinini 25357 (CTES). Jujuy, dep. Gral. San Martín, Parque Nacional Calilegua, camping Aguas Negras, 17-IV-98, Ferraro et al. 5919 (CTES).

Trichothelium ake-assii Becker \& Lücking (Trichotheliales: Trichotheliaceae)

Fig. 3, C 


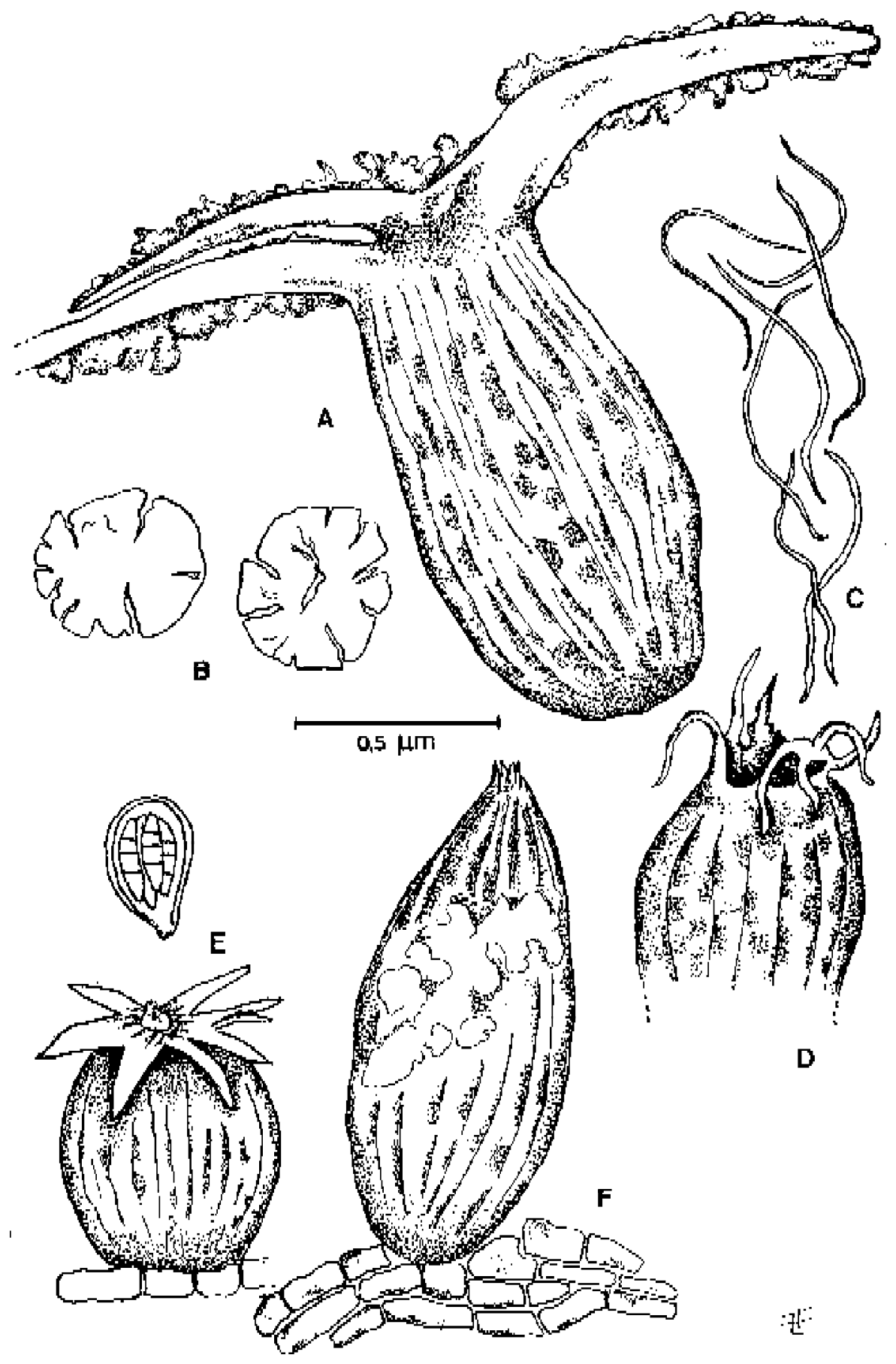

Fig. 4: A-D, Lyromma nectandrae (Popoff 2896, CTES), A: picnidios, B: cristales de la base de los conidios, C: conidios, D: ápice de un conidio. E: Lyromma ornata (Ferraro 5916 CTES), peritecios y ascas con esporas. F: Lyromma palmae (Ferraro 5916, pp., CTES) conidio con picnidios y algas Phycopeltis en la base. 
Presenta peritecios poco elevados y con base ancha, robustos, coronados de setas. Las setas forman manojos muy conspicuos y oscuros cerca del ostíolo pero de color más claro en los ápices que se adelgazan. Las esporas tienen 8-11 septos. Ampliamente distribuido en selvas lluviosas tropicales pero no común.

Material estudiado: ARGENTINA. Misiones, dep. Iguazú, Parque Nacional Iguazú, Sendero Macuco, sobre hojas de Rutaceae, 28-III-95, Ferraro et al. 5005 (CTES); ídem, Ferraro et al. 5015 (CTES).

Trichothelium bipindense F. Schill. Fig. 3, D

Con peritecios y setas negras, esporas con 9-11 septos, algunas hasta 12-septadas, anchas. Esta especie fue previamente registrada para Brasil (Ferraro, 1997) y Costa Rica (Lücking, 1998).

Material estudiado: ARGENTINA. Misiones, dep. Iguazú, Parque Nacional Iguazú, Sendero Macuco, sobre hojas de Rutaceae, Ferraro 5004 (CTES); ídem, Ferraro 5027 (CTES); ídem, sendero Yacaratia, sobre Rhapanea, Ferraro et al. 5074 (CTES).

\section{Trichothelium minus Vain.}

Caracterizado por peritecios negros, pequeños, cubiertos por ca. de 4-10 setas con base anchas y muy delgadas en los extremos, esporas 3-5 septadas, 21-25 um de longitud. Común en Misiones, era conocido de Brasil, (Santesson, 1952); Aptroot \& Sipman (1993) lo consideran neotropical y mencionan la especie para Guiana y Guatemala.

Material estudiado: ARGENTINA. Misiones, dep. Gral. Belgrano, Colonia Andresito, Chacra 30, sobre hojas de Sorocea, 1-III-96, Vanni et al. 3670 (CTES). BRASIL. Estado de Santa Catarina, Foz de Iguazú, Parque en el área de Cataratas del Iguazú, sobre hojas de Sorocea, 1006-98, Ferraro 5890 (CTES, hb. Lücking).
Trichothelium minutum (R. Lücking) R. Lücking

Puede identificarse por las setas muy largas y angostas, escasas, con los extremos aguzados claros, blanquecinos y curvadas hacia abajo (deflexas), esporas 7 septadas. Fue descrita para Costa Rica y mencionada por Aptroot y Sipman (1993) para Guiana Francesa, nuestra colección amplía el área de distribución conocida de la especie.

Material estudiado: ARGENTINA. Misiones, dep. Iguazú, Parque Nacional Iguazú, ruta 101, 8-VIII-95, Maruñak et al. 869 (CTES).

\section{Agradecimientos:}

Agradecemos al Dr. Vobis de la Universidad Nacional del Comahue (Bariloche, Argentina), las sugerencias brindadas para la obtención de cortes microscópicos, sobre los que estudiamos la morfología interna de los taxones, también la realización de algunas fotografías

\section{Bibliografía}

Aptroot, A \& H. J. M. Sipman (1993) Trichotheliaceae. In: GÖRTS-VAN RIJN, A.R.A. (Ed.): Flora of the Guianas, 2:1-57. Koeltz, Königstein.

Cáceres, M. E. da S., Maia, L. C. \& Lücking, R. (2000) Foliicolous lichens and their lichenicolous fungi in the Atlantic rainforest of Brazil: Diversity, ecogeography and conservation. Bibliotheca Lichenologica 75 (en prensa).

Cabrera, A. L. (1971) Fitogeografía de la República Argentina. Bol. Soc. Arg. Bot. 24(1-2): 142, 8 figs.

Cavalcante, W. de A. \& A. A. Silva in Cavalcante et al., Publcões Inst. Micol. Recife, 647: 37 (1972).

Farkas, E. \& H. J. M. Sipman (1997) Checklist of foliicolous lichenized fungi. Abstr. Bot. 21(1): 173-206.

Ferraro, L. I. (1997) Checklist of foliicolous lichenized fungi from North-Eastern Argentina (Corrientes and Misiones), with notes on adjacent areas of Paraguay and Brasil. Abstr. Bot. 21(1): 53-63. 
Ferraro, L. I. \& R. Lücking(1999). New species or interesting records of foliicolous lichens. V. Two new species of Fellhanera (Lichenized Ascomycotina: Pilocarpaceae) with 1septate ascospores. Mycotaxon 73: 163-167.

Lücking, R. (1992) Foliicolous lichens. A contribution to the knowledge of the lichen flora of Costa Rica, central America. Beih. Nova Hedwigia 104:1-179.

Lücking, R. (1997) Additions and corrections to the knowledge of the foliicolous lichen flora of Costa Rica. The family Gomphillaceae. Bibl. Lichenol. 65:1-109, 18 plates. Cramer, Berlin, Stuttgart.

Lücking, R. (1998) Additions and corrections to the knowledge of the foliicolous lichen flora of Costa Rica. The genus Trichothelium (lichenized Ascomycetes:Trichotheliaceae). Nova Hedwigia 66: 375-417.

Lücking, R. (1999) Foliicolous lichens and their lichenicolous fungi from Ecuador, with a comparison of lowland and montane rain forest. Willdenowia 29: 299-335.

Lücking, R. \& Kalb, K. (2000) Foliikole Flechten aus Brasilien (vornehmlich Amazonien), inklusive einer Checkliste und Bemerkungen zu Coenogonium und Dimerella (Gyalectaceae). Botanische Jahrbücher für Systematik 122: 1-61.

Lücking, R., E. Sérusiaux, E. Maia L. C. \& E. C. G. Pereira (1998). A revision of the names of foliicolous lichenized fungi published by Batista and co-workers between 1960 and 1975. Lichenologist 30: 121-191.

Lücking, R., Farkas, E. , Sérusiaux, E. \& Sipman, H. J. M. (2000) Checklist of foliicolous lichens and their lichenicolous fungi. Part I. Foliicolous lichens. [http:// www.unibayreuth.de/departments/ planta2/ ass/robert/lichens/checkfol.html]

Matzer, M. (1996) Lichenicolous Ascomycetes with fissitunicate asci on foliicolous lichens. Myc. Pap. 171: i-x, 1-202.

Santesson, R. (1952) Foliicolous Lichens. I. Sym. Bot. Ups. 12: 1-590.

Sérusiaux, E. (1983) Foliicolous lichens from Zimbabwe. Lichenol. 15(3): 283-287.

Sérusiaux, E. (1989) Foliicolous lichens: ecological and chorological data. Botanical Journal of the Linnean Society 100: 87-96.

Sérusiaux, E. (1992) Reinstatement of the lichenized genus Eremothecella Sydow. Systema Ascomycetum 11(1): 39-47. 